\title{
AN AUTOMATIC STABILIZED DETECTION SYSTEM FOR MEASURING SOFT CELESTIAL X-RAYS
}

\author{
A. J. F. DEN BOGGENDE, H. F. VAN BEEK, A. C. BRINKMAN, \\ and $\mathrm{H}$. Th. J. A. LAFLEUR \\ Space Research Laboratory, Utrecht, The Netherlands
}

In order to observe celestial X-ray sources an instrument is under development to be launched in the Astronomical Netherlands Satellite (ANS) in 1974. The aim of the experiment is to measure the spectral distribution of the sources. If there is some evidence that a source should be a pulsar the instrument can be switched from the normal mode to the pulsar mode. In this latter mode the detected photons are labeled in time using an on board clock. The ANS will have a pointing mode and a slow scanning mode. The maximum observing time per orbit for one object will be about $2000 \mathrm{~s}$.

The.instrument as a whole consists of two systems, each having a collimating part and a detector. One system makes use of a proportional counter with a $2 \mu$ titanium window of about $150 \mathrm{~cm}^{2}$, sensitive to radiation between $1-11 \AA$ and $27-35 \AA$. The 1-11 $\AA$ region is electronically subdivided into five channels. In front of this detector a honeycomb collimator is placed with a viewing angle of $1^{\circ}$ FWHM. The other detection system uses a proportional counter with a 6 micron polypropylene window (covered with $1000 \AA$ aluminum) of about $7 \mathrm{~cm}^{2}$. The detector is sensitive to radiation between 1-15 $\AA$ and 44-60 $\AA$. A grazing incidence parabolic mirror is placed in front of the window. The mirror has a collecting area of $144 \mathrm{~cm}^{2}$ and a viewing angle of $20^{\prime}$ FWHM. The reflectivity for radiation of $\lambda>30 \AA$ will be at least $50 \%$.

Both proportional counters mentioned above are composed of different sections, each of them being a counter in itself, separated by means of cathode wires. These main counters are surrounded partly by anti-coincidence counters also separated from the main counters by means of cathode wires.

The anti-coincidence counters will generate anti-coincidence pulses for all cosmic particles and relatively high energy electrons ejected from the counter wall by gamma radiation or Bremsstrahlung. Therefore no pulse shape discrimination is needed. The background component caused by low energy electrons ejected from the counter walls, will be reduced strongly, since the detector described fulfils the walless counter concept very closely. This background component cannot be suppressed by means of pulse shape discrimination.

One of the anti-coincidence counters of each detector will be continuously irradiated by a ${ }^{55} \mathrm{Fe}$ radioactive source, emitting $2.1 \AA$ radiation. The pulses from these counters serve after pulse height discrimination as control signals to adjust the high voltage supplies in order to maintain a constant gas gain during a long time. This feedback system operates within certain limits.

If too much gas leaked out of the counters no further high voltage adjustment is

Labuhn and Lüst eds. ., New Techniques in Sinace Astronomy, 211 212.

All Rights Reserved. Copiright C 1971 by the IAL 
possible. Therefore a gas supply system has been incorporated for each detector providing the possibility to add a known amount of gas to the counters. Each system consists of a gas bottle, two solenoide valves and an intermediate reservoir, and will be operated on ground command.

Inflight calibration will be performed by means of radioactive sources $\left({ }^{55} \mathrm{Fe}\right)$, normally covered by shutters.

A shutter mechanism is available to check the reflectivity of the parabolic mirror using the radiation of a strong celestial source.

\section{DISCUSSION}

D. Hovestadt: Is there anything known about the mechanism which reduces the gas gain in a methane counter?

A. J. F. den Boggende: Yes, there is a paper in J. Sci. Inst. J. Phys. E 1969, p. 701 written by me where some results are given and suggestions about the mechanism.

$R$. Novick: With $\mathrm{CO}_{2}$ as quench gas we find a very long life time. Irradiating a counter with $6 \mathrm{keV}$ radiation with a count-rate of 8000 photons/s we find very little change in gas gain (less than $3 \%$ ) and no change in resolution (less than $0.5 \%$ for $6 \mathrm{keV}$ radiation). $\mathrm{CH}_{4}$ gives a very short life time with the same test.

H. Lafleur: The use of a pressure regulator excludes the possibility of easily changing the gas pressure. 